\title{
Influence of body mass index and gender on physical activity in primary school children during PE and non-PE school days
}

\author{
D.T. Archer, B. Hogg, M. Coulson, I. Soos, S.D. Anderson, P. Innerd and S.D. Leyland \\ School of Nursing and Health Sciences, University of Sunderland, Sunderland SR1 3SD, UK.
}

The benefits of physical activity (PA) for children are well recognised. Children who display high levels of moderate to vigorous physical activity (MVPA) have a lower risk of becoming overweight/obese ${ }^{(1)}$ or becoming inactive adults ${ }^{(2)}$. Existing evidence suggests a potential interaction between gender and BMI in terms of PA, with low MVPA associated with those with greater BMI z-scores in 7-9 year old girls, but not boys ${ }^{(3)}$. In 2014, the new National Curriculum for England was implemented across the UK for Physical Education (PE). The aim of this study was to compare objectively measured PA on PE and non-PE days and determine whether children's BMI and gender influenced the differences found.

PA was measured using tri-axial accelerometers (Actigraph GT3X-BT) worn at the hip in male $(n$ 47) and female $(n$ 39) children aged 6.8 (SD 0.6) years, height 123.2 (SD 5.6) $\mathrm{cm}$ and body mass 24.45 (SD 3.95) $\mathrm{kg}$ over four days at school (360 min per day). The duration of sedentary and MVPA in regular class time and structured PE classes (46 (SD 12) min duration) was measured using the previously validated Pulsford cut off points ${ }^{(4)}$. BMI z-scores were calculated using WHO guidelines. Data were analysed using Pearson correlations, paired samples and independent samples $t$-tests, Cohen's d for effect sizes (ES) and are presented as mean (SD).

\begin{tabular}{|c|c|c|c|c|c|c|c|c|}
\hline & \multicolumn{2}{|c|}{ PE class } & \multicolumn{2}{|c|}{ non-PE class } & \multicolumn{2}{|c|}{ PE days } & \multicolumn{2}{|c|}{ non-PE days } \\
\hline & Mean & SD & Mean & SD & Mean & SD & Mean & SD \\
\hline$\overline{M V P A ~(m i n) ~}$ & $13 \cdot 3$ & $4 \cdot 0$ & $3 \cdot 0^{*}$ & 1.5 & $41 \cdot 5$ & $10 \cdot 2$ & $34 \cdot 0^{*}$ & 9.0 \\
\hline Sedentary (min) & $15 \cdot 6$ & $6 \cdot 6$ & $31.4 *$ & 9.4 & $218 \cdot 5$ & $21 \cdot 1$ & $232.9 *$ & $18 \cdot 3$ \\
\hline
\end{tabular}

Children carried out more MVPA on PE days compared to non-PE days $(\mathrm{ES}=0.78)$ and during PE lessons compared to corresponding non-PE time. Furthermore, children were significantly less sedentary in PE lessons compared to corresponding non-PE time. Consequently, children spent less overall time in the sedentary activity category on PE days compared to non-PE days $(\mathrm{ES}=0 \cdot 68)$. Childrens' BMI z-scores correlated significantly with their quantity of sedentary and light activity $(\mathrm{P}<0 \cdot 001)$, but not MVPA $(\mathrm{P}=0.60)$ and were very similar on both $\mathrm{PE}$ and non-PE days. Males were more active than their female peers, performing 8 and 6 min more MVPA (ES 0.89, 0.72) and 12 and 8 min less sedentary time on PE and non-PE days (ES -0.62, -0.42), respectively.

In conclusion, PE lessons increased childrens' daily MVPA and reduced their sedentary time. Although gender and BMI were associated with PA levels, the inclusion of PE lessons did not markedly increase their influence on overall energy expenditure. Overall, the study shows that PE lessons have a positive effect on childrens' PA levels, likely resulting in positive effects on adiposity and overall health and wellbeing.

1. Bornstein D, Beets M, Byun W et al. (2011) J Sci Med Sport 14, 504-511.

2. Biddle SJH, Pearson N, Ross GM et al. (2010) Prev Med 51, 345-51.

3. Basterfield L, Adamson AJ, Pearce MS et al. (2011) J Phys Act Health 8, 543-547.

4. Pulsford RM, Cortina-Borja M, Rich C et al. (2011) PLoS One 6, 1-9. 